\title{
Too much and not enough: revisiting maintenance rituximab in indolent lymphomas
}

\author{
Sonali M. Smith \\ Section of Hematology/Oncology, University of Chicago, Chicago, IL, USA \\ E-mail: SONALI M. SMITH - smsmith@medicine.bsd.uchicago.edu
}

doi:10.3324/haematol.2021.279101

$\mathrm{D}$ espite being slow-growing and chemosensitive diseases, the inevitable outcome of indolent lymphomas is relapse and prevention of relapse has been an important avenue of investigation for decades. The advent of rituximab allowed consideration of a less toxic (compared to chemotherapy) opportunity to maintain remission following induction regimens without the need for prolonged exposure to cytotoxic agents. The common questions have focused on both the schedule and the duration of maintenance therapy, with the goal to improve progressionfree or overall survival; in an ideal world, maintenance strategies might even seek to cure.

Rituximab pharmacokinetics and/or impact on B-cell depletion and subsequent recovery provide sufficient rationale for delivering maintenance rituximab with a variety of approaches: one dose every 8 weeks, one dose every 12 weeks, or four weekly doses every 6 months. ${ }^{1,2}$ Although none of these schedules has been directly compared, small retrospective reviews suggest relative equivalence in terms of efficacy and small differences in terms of toxicity. ${ }^{3}$ In frontline follicular lymphoma, the PRIMA trial established one dose of maintenance rituximab every 8 weeks based on achieving a trough level of $25 \mu \mathrm{g} / \mathrm{mL}$ in the majority of patients, and this has arguably become the most common schedule. ${ }^{4}$ It is important to note that there remains a lack of a survival advantage for maintenance rituximab in frontline follicular lymphoma, but 10-year data show impressive and persistent disease control and validate maintenance rituximab for 2 years as an appropriate option to improve progression-free survival in patients with high-tumor burden follicular lymphoma.

While the schedule of rituximab maintenance can be justified based on pharmacokinetics, the duration of rituximab maintenance is more empirically derived. Given the relatively favorable toxicity profile even with prolonged administration, studies have ranged from several limited doses to 5 years of treatment to indefinite treatment. In the relapsed setting, a meta-analysis from a decade ago suggested improved overall survival for maintenance rituximab in relapsed/refractory follicular lymphoma, although it is critical to acknowledge that the majority of trials included in this study had involved chemotherapy induction and not chemoimmunotherapy induction. ${ }^{5}$

Based on improved progression-free survival and a lure of improved overall survival, is more maintenance better? In this issue of Haematologica, ${ }^{6}$ Rule and colleagues present the final results of the MabCute trial. This prospective, international, randomized phase III trial sought to determine the added benefit of extended rituximab dosing beyond 2 years in responding patients with indolent lymphomas. All patients had relapsed or refractory indolent lymphomas, and could receive any chemoimmunotherapy induction followed by 2 years of rituximab maintenance. Using 2007 response criteria, responding patients at 2 years were randomized to receive either an additional 2 years of maintenance therapy with subcutaneous rituximab or active observation. With a primary endpoint of progression-free survival, 274 patients were randomized and the median follow-up is 28 months. Adverse events were slightly increased in the extended maintenance arm. During the observation period, the number of progression events was quite low in both arms, and the trial is now closed without a clear signal of improved progression-free survival for the extended maintenance arm; no conclusions can be made regarding survival.

The MabCute trial can thus be added to the list of trials showing no advantage from prolonged maintenance with anti-CD20 targeting strategies if disease control, toxicity, and overall survival are considered collectively. As one example, the SAKK 35/03 trial randomized a heterogeneous group of patients with follicular lymphoma to receive either rituximab every other month for four administrations or rituximab every other month for 5 years; ${ }^{7}$ patients in this trial could have had treatment-naïve, relapsed, or refractory disease and all received induction therapy with rituximab monotherapy and not chemoimmunotherapy. While event-free survival was improved, there was more toxicity and no impact on survival in the prolonged treatment arm. Other key trials have shown no benefit from rituximab treatment at relapse compared to a maintenance approach $;^{8}$ specifically, the RESORT trial found no difference in time to next treatment and no improvement in overall survival between maintenance and retreatment in low tumor burden indolent lymphomas. Finally, the induction chemotherapy backbone also influences the risk-benefit profile; for example, the GALLIUM trial observed that patients receiving bendamustinebased induction had more toxicity and even increased mortality, particularly during the maintenance component of therapy. ${ }^{9}$ It is worth noting that the majority of patients in the current trial also received bendamustine-based regimens and no toxicity signal was seen with the extended maintenance, but this may be because of drop-out during the initial maintenance component.

The COVID-19 pandemic has forced us to re-evaluate data with an additional critical lens related to treatment-associated B-cell suppression. Early studies during this pandemic showed that patients on immune suppression or those on chemotherapy suffer more severe complications related to SARS-CoV2, and mortality in patients with hematologic malignancies is high. ${ }^{10}$ Since maintenance rituximab has yet to offer cure or improved overall survival after chemoimmunotherapy induction, it seems more appropriate to identify the minimum number of doses rather than trying to expand or extend treatment. Indeed, only a minority of patients with blood cancers mount a sufficient response to vaccines; ${ }^{11}$ accordingly, recent scientific society (https://www. hematology.org/covid-19/ash-astct-covid-19-andvaccines) and advocacy guidelines ${ }^{12}$ highlight that patients receiving B-cell-directed therapies have attenuated or even 
absent responses to vaccination against SARS-CoV2 and cannot abandon social distancing and masking precautions.

In the end, there is no advantage from prolonged maintenance in indolent lymphomas, and 2 years should remain the standard duration if maintenance rituximab is offered. The MabCute trial thus supports that extended dosing of rituximab is both too much, and also not enough to offer cure or improve overall survival. Future studies should focus on identifying the minimum number of maintenance doses needed to improve outcomes, particularly in light of a pandemic threat.

\section{Disclosures}

No conflicts of interest to disclose.

\section{References}

1. Berinstein NL, Grillo-Lopez AJ, White CA, et al. Association of serum rituximab (IDEC-C2B8) concentration and anti-tumor response in the treatment of recurrent low-grade or follicular non-Hodgkin's lymphoma. Ann Oncol. 1998;9(9):995-1001.

2. Gordan LN, Grow WB, Pusateri A, Douglas V, Mendenhall NP, Lynch JW. Phase II trial of individualized rituximab dosing for patients with CD20-positive lymphoproliferative disorders. J Clin Oncol. 2005;23(6):1096-1102.

3. Nabhan C, Ollberding NJ, Villines D, et al. A systematic review of comparative schedule-related toxicities with maintenance rituximab in follicular and mantle cell lymphomas. Leuk Lymphoma. 2014;55(6):1288-1294.
4. Salles G, Seymour JF, Offner F, et al. Rituximab maintenance for 2 years in patients with high tumour burden follicular lymphoma responding to rituximab plus chemotherapy (PRIMA): a phase 3, randomised controlled trial. Lancet. 2011;377(9759):42-51.

5. Vidal L, Gafter-Gvili A, Salles G, et al. Rituximab maintenance for the treatment of patients with follicular lymphoma: an updated systematic review and meta-analysis of randomized trials. J Natl Cancer Inst. 2011;103(23):1799-1806.

6. Rule S, Barreto W, Briones J, et al. Efficacy and safety assessment of prolonged maintenance with subcutaneous rituximab in patients with relapsed or refractory indolent non-Hodgkin lymphoma: results of the phase III MabCute study. Haematologica. 2021;107(2):500-509.

7. Taverna C, Martinelli G, Hitz F, et al. Rituximab maintenance for a maximum of 5 years after single-agent rituximab induction in follicular lymphoma: results of the randomized controlled phase III trial SAKK 35/03. J Clin Oncol. 2016;34(5):495-500.

8. Kahl BS, Hong F, Williams ME, et al. Rituximab extended schedule or re-treatment trial for low-tumor burden follicular lymphoma: Eastern Cooperative Oncology Group protocol E4402. J Clin Oncol. 2014;32(28):3096-3102.

9. Hiddemann W, Barbui AM, Canales MA, et al. Immunochemotherapy with obinutuzumab or rituximab for previously untreated follicular lymphoma in the GALLIUM study: influence of chemotherapy on efficacy and safety. J Clin Oncol. 2018;36(23):2395-2404.

10. Mato AR, Roeker LE, Lamanna N, et al. Outcomes of COVID-19 in patients with CLL: a multicenter international experience. Blood. 2020;136(10):1134-1143.

11. Herishanu Y, Avivi I, Aharon A, et al. Efficacy of the BNT162b2 mRNA COVID-19 vaccine in patients with chronic lymphocytic leukemia. Blood. 2021;137(23):3165-3173.

12. Proceedings of the COVID-19 Vaccine Panel: a Lymphoma Research Foundation White Paper. https://lymphoma.org/wpcontent/uploads/2021/04/Proceedings-of-the-COVID-19-VaccinePanel_LRF-White-Paper_April-2021.pdf; 2021. (Last accessed May 12, 2021)

\section{An exciting RXRA mutant revives interest in retinoids for acute myeloid leukemia}

\section{Fang Qiu' and Hugues De The ${ }^{1-3}$}

${ }^{1} I N S E R M ~ U M R$ 944, CNRS UMR 7212, Université de Paris, IRSL, Hôpital Saint Louis; ${ }^{2}$ Collège de France, Oncologie Cellulaire et Moléculaire, PSL University, INSERM UMR 1050, CNRS UMR 7241, and ' ${ }^{3}$ Department of Hematology, Hôpital Saint Louis (Assistance publique Hôpitaux de Paris) and Paris University, Paris, France

E-mail: HUGUES DE THÉ - hugues.dethe@inserm.fr

doi:10.3324/haematol.2021.279152

T he idea that "one size fits all" is obviously outdated for acute myeloid leukemia (AML) therapy: tomorrow's treatments will depend on phenotypically or genetically defined subtypes. The most striking example is acute promyelocytic leukemia (APL), driven by the PMLRARA fusion protein. In APL, a subtype that accounts for $5 \%$ of cases of AML, a combination of two targeted agents, all trans-retinoic acid (ATRA) and arsenic trioxide, cures over $90 \%$ of patients through PML-RARA driver degradation, differentiation and restoration of PML-dependent senescence. ${ }^{1}$ These clinical successes have spurred attempts to harness the power of retinoids in other cancers. Unfortunately, ATRA treatment alone remains poorly effective in most non-APL AML. ${ }^{2}$

Retinoid signaling is complex and still incompletely understood. ${ }^{3}$ ATRA acts primarily through heterodimeric complexes of retinoic acid receptors (RAR) assembled with retinoid X receptors (RXR). These RXR are key heterodimerization partners of many class-II nuclear receptors and may be ligand-dependent transcription factors or silent receptors, allowing sequence-specific DNA recognition. ${ }^{4}$ Hence, therapeutic targeting of RXR could be a strategy to activate targets under the control of the RXR/RAR transcriptional complex. However, in principle, RXR/RAR signaling cannot be activated by RXR ligands alone, at least in part because co-repressors remain firmly bound to RAR. This may be modulated by other signaling cascades/second messengers, such as cAMP. ${ }^{5}$

In AML, this simple view has been challenged. RXR ligands (rexinoids, such as bexarotene) may exert some differentiating effects ex vivo and in vivo, 5,6 Hematopoietic cells and some AML express endogenous RXRA ligands. ${ }^{7,8}$ Two recent studies have revived interest in RXRA signaling in AML. The first demonstrated that, in AML driven by KMT2A-MLLT3, rexinoids partially suppressed AML growth and triggered differentiation. ${ }^{8}$ Moreover, genetic ablation of RXR accelerated AML growth, while concomitant activation of both RXRA and RARA precipitated differentiation or apoptosis. It is hoped that dual activation of these key regulators may harness retinoids more effi- 